\title{
Pulmonary Hydatid Cysts Masquareading As Aspergilloma Lung: A Case Report
}

\author{
Mangilal Choudhary, Dilip Ramrakhiani, Karuna Gupta, Ajay Yadav, Maneesh Vijay \\ Department of Pathology, SMS Medical College, Jaipur, Rajasthan, India.
}

\begin{abstract}
A 25-year-old female presented with cough, hemoptysis and low grade fever of one-month duration. There was no history of pulmonary disease or immunosuppression. A chest radiograph show segmental consolidation in upper lobe of right lung. Computerized tomography (CT) scan revealed $5.2 \times 4.7 \mathrm{~cm}$ sized round to oval cavitary lesion with air crescent in right upper lobe associated surrounding tiny centrilobular nodule and peribronchial thickening. Possibility of secondary aspergillosis in a preexisting tuberculous cavity. Sputum tests for acid fast bacilli, bacteria and fungi, were all negative. The patient underwent resection of a upper lobe of right lung, and a wedge resection specimen was sent for histopathological examination in the department of pathology. Grossly a lung lobectomy specimen ms. $10 \times 6 \times 5 \mathrm{~cm}$. On c/s cyst identified ms. $5 \times 4$ $\mathrm{cm}$. in diameter was identified, it was unilocular white laminated and filled with clear fluid. Subsequent microscopic examination demonstrated that acellular laminated hyaline material (ectocyst), while surrounding
\end{abstract}

\section{INTRODUCTION}

Infection by Aspergillus is usually observed in immunocompromised patients while hydatid disease infected by tapeworm Echinococcus is endemic in many parts of the world, most commonly in Middle East, Australia, Iceland, and South America. Liver and lungs are often affected. ${ }^{1-5}$ But Aspergillus within a hydatid cyst is extremely rare. Patients reported mostly were in middle-aged or suffering from other diseases. Patient in this case is youngest one ever reported and is immunocompetent. In this case we described clinical, radiological correlation \& pathological manifestation by possible mechanisms for development of hydatid cyst in lungs.

\section{CASE HISTORY}

A 25-year-old female presented with cough, hemoptysis and low grade fever since one-month duration. Patient was from Pali marwar, an area of Rajasthan where hydatid disease is fairly common because people keep domestic animals. There was no history of pulmonary disease or immunosuppression. Sputum tests for acid fast bacilli, bacteria and fungi were all negative. Hydatid serology by enzyme linked immunosorbent assay (ELISA) was weakly positive for serum Echinococcal antibodies (lgGs). Results of other laboratory tests such as hemogram, routine lung parenchyma showed dense inflammatory infiltrate comprised of lymphocytes \& giant cells, features consistent with hydatid cyst disease.

Keywords: Hydatid Cyst, Aspergillosis, Hemoptysis.

\section{*Correspondence to:}

\section{Dr. Mangilal Choudhary}

Resident,

Department of Pathology,

SMS Medical College,

Jaipur, Rajasthan, India.

Article History:

Received: 16-09-2016, Revised: 02-10-2016, Accepted: 21-10-2016

\begin{tabular}{|l|r|}
\hline \multicolumn{2}{|c|}{ Access this article online } \\
\hline Website: & Quick Response code \\
www.ijmrp.com & \\
\hline DOI: & \\
10.21276/ijmrp.2016.2.6.046 & \\
\hline
\end{tabular}

urinalysis, stool examination, and tests of liver function did not reveal any abnormalities. The patient was also negative for human immunodeficiency virus. Abdominal ultrasonography had shown no liver lesions. Dyskaryotic cells were found by bronchoscopic brush biopsy.

\section{DISCUSSION}

A fully developed hydatid cyst consists of three layers, an inner germinal layer (endocyst), an outer laminated layer (ectocyst) and a host-derived fibrous layer (pericyst). From germinal layer, scolices, brood capsules, and daughter cysts develop. Clinical manifestation of pulmonary hydatid disease depends on whether cyst remains intact. Intact cysts remain asymptomatic or cause mild symptoms such as cough, dyspnoea, and chest pain.

In addition, patients with a complicated cyst may discharge contents of cyst, and present with hemoptysis or fever. On a chest plain film or CT, intact cysts may display as round or oval masses that possess well-defined borders. Immune state of patient and structural alterations in lung may predispose to Aspergillus infection.

Generally, invasive pulmonary aspergillosis is observed in severely immunocompromised patient, chronic necrotizing 
aspergillosis in mildly immunocompromised, and aspergilloma in patients with existing lung cavities. Most common symptoms are low fever, chronic productive cough and hemoptysis. In this report, cough, hemoptysis, and fever were patient's chief complaints, and a solitary pulmonary mass in right lung was discovered. Diagnosis of hydatid cyst was made by chest CT, and consistent with serology (serum Echinococcal antibodies).

To avoid risk of anaphylaxis and dissemination, patient underwent surgery. Aspergillosis and echinococcosis share same symptoms

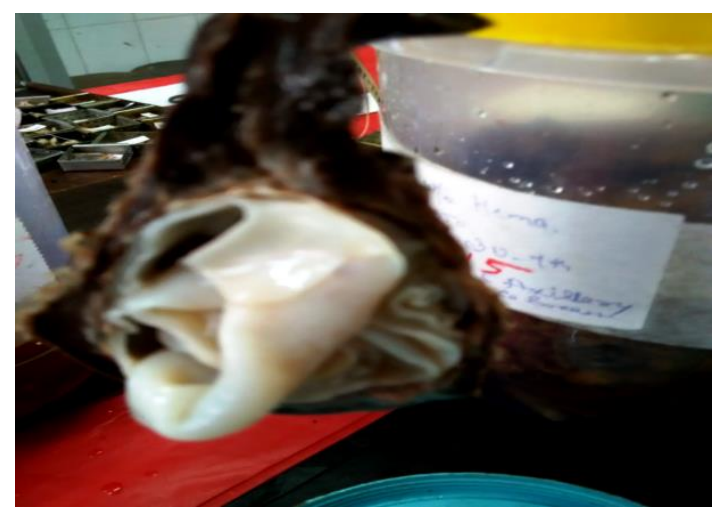

and crescent signs on chest CT making difficult to distinguish. In this case, symptoms were not result of hydatid cyst rupture because gross pathology showed hydatid cysts intact. Even if cyst were burst, and there was nothing to discharge.

So we assume that symptoms of patient were mainly caused by Aspergillus infection, but this hypothesis requires further confirmation. Hydatid cysts containing fungi resembling Aspergillus are extremely rare. At present, such cases have been uncommonly reported; there are no enough data to further study.

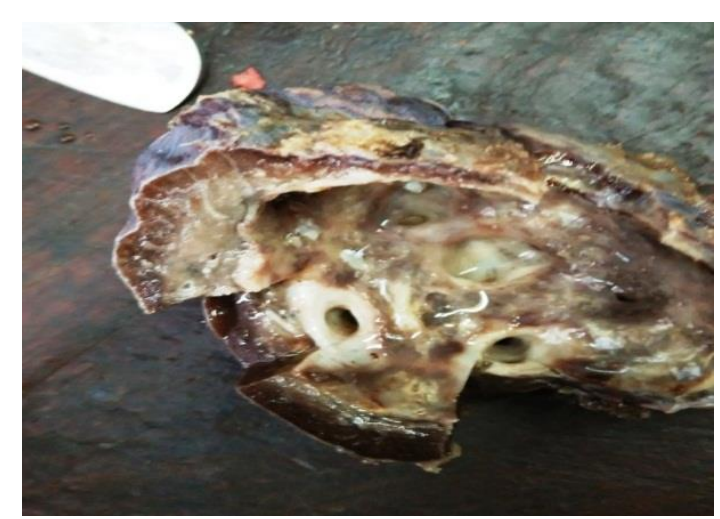

Fig 1,2: Gross morphology: A lung lobectomy specimen $\mathrm{ms} .10 \times 6 \times 5 \mathrm{~cm}$. On C/S cyst is identified ms. $5 \times 4 \mathrm{~cm}$ in diameter, it was unilocular white laminated hyaline membrane and filled with clear fluid.

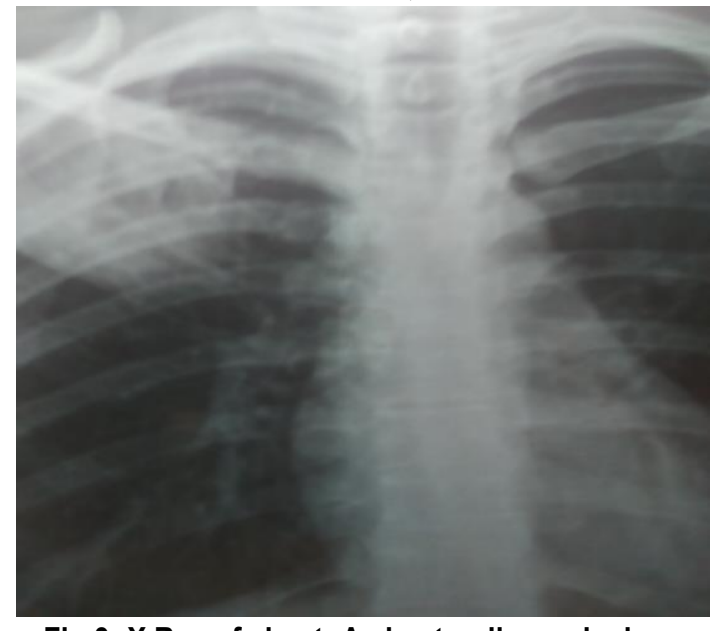

Fig 3: X Ray of chest: A chest radiograph show segmental consolidation in upper lobe of right lung.

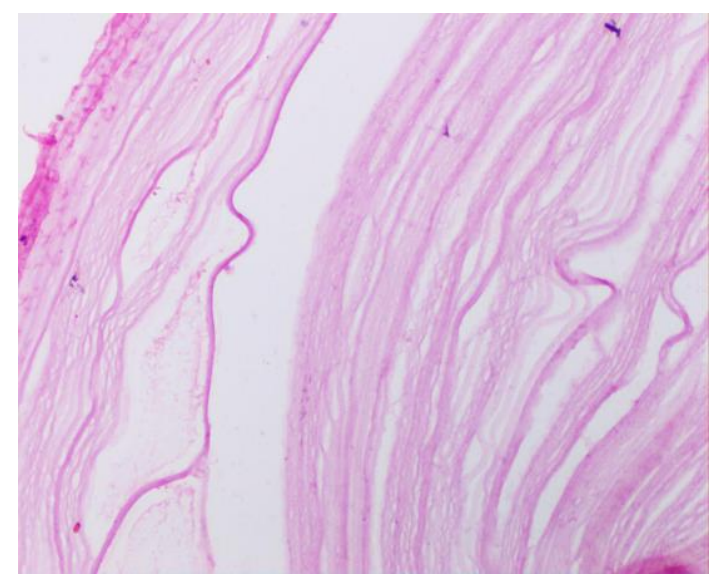

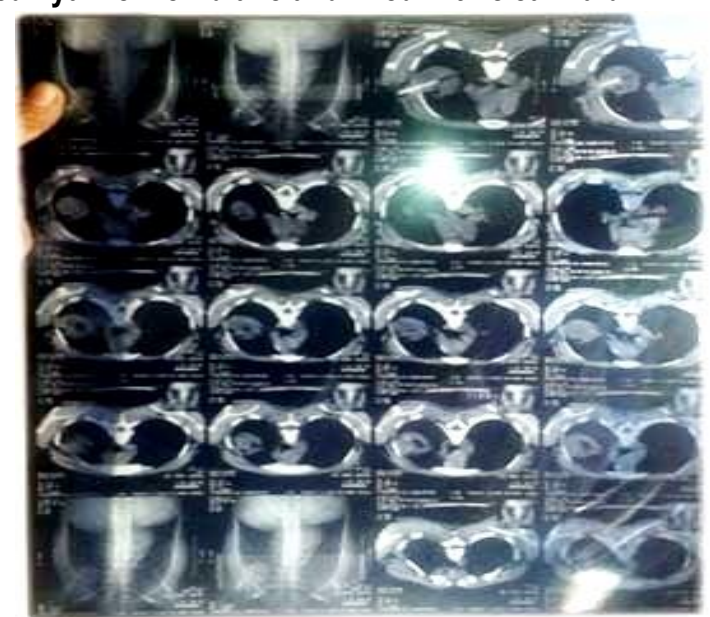

Fig 4: CT Thorax (plain \& contrast) $-52 \times 47 \mathrm{~mm}$ sized round to oval cavitary lesion with air cresent in Right upper lobe associated surrounding tiny centrilobular nodules and peribronchial thickening. Possibility of secondary aspergillosis in a preexisting tuberculous cavity is more likely than primary fungal infection.

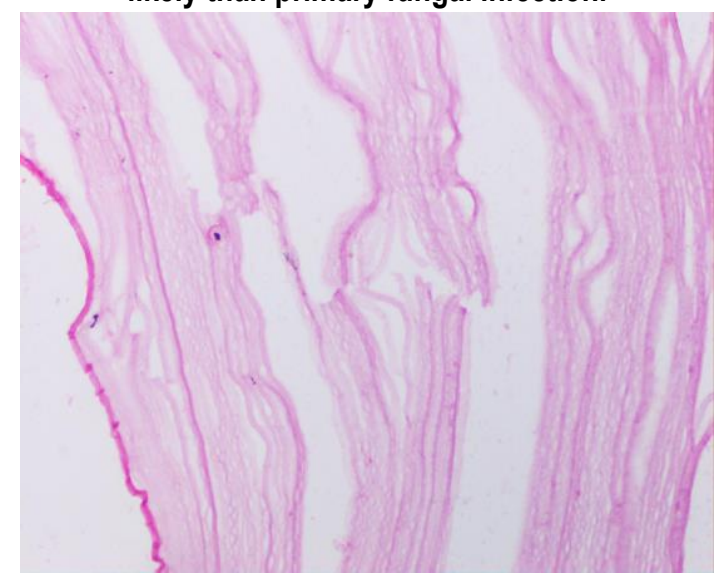

Fig 5,6: Microscopic Morphology: Multiple sections examined revealed layer composed of acellular laminated hyaline material (ectocyst) while surrounding lung parenchyma showed dense inflammatory infiltrate comprise of lymphocytes \& giant cells. These histomorphological features favour Hyadatid cyst disease. 


\section{CONCLUSSION}

Aspergillosis and echinococcosis share same symptoms and crescent sign on CT making it difficult to distinguish. In a patient clinically and radiologically suspected as aspergilloma, a differential of hydatid cyst should be kept in mind. In this case report we have described clinicoradiological correlation and pathological manifestation and possible mechanism for development of hydatid cyst in lungs. Both aspergillosis and echinococcosis can coexist as cited in references.

\section{REFERENCES}

1. Bal $A$, Bagai $M$, Mohan $H$, et al. Aspergilloma in a pulmonary hydatid cyst: a case report. Mycoses 2008;51:357-9.

2. Koçer NE, Kibar Y, Güldür ME, et al. A retrospective study on the coexistence of hydatid cyst and aspergillosis. Int J Infect Dis 2008;12:248-51.

3. Vaideeswar P, Vyas M, Katewa A, et al. Piggyback mycosis: pulmonary hydatid cyst with a mycotic co-infection. Mycoses 2010;53:265-8.

4. Chu XD, Wang GZ, Feng $X H$, et al. Risk factors on human cystic echinococcosis in HobukesarMongolian Autonomous County in Xinjiang. Zhonghua Liu Xing Bing Xue Za Zhi 2010;31:297-9.
5. Jin Bing Ban, Yu Hong et al . A case report of hydatid cyst containg aspergillosis . 2012, issn.2072-1439.2012.07.04 [

\section{Source of Support: Nil.}

Conflict of Interest: None Declared.

Copyright: () the author(s) and publisher. IJMRP is an official publication of Ibn Sina Academy of Medieval Medicine \& Sciences, registered in 2001 under Indian Trusts Act, 1882.

This is an open access article distributed under the terms of the Creative Commons Attribution Non-commercial License, which permits unrestricted non-commercial use, distribution, and reproduction in any medium, provided the original work is properly cited.

Cite this article as: Mangilal Choudhary, Dilip Ramrakhiani, Karuna Gupta, Ajay Yadav, Maneesh Vijay. Pulmonary Hydatid Cysts Masquareading As Aspergilloma Lung: A Case Report. Int J Med Res Prof. 2016; 2(6):227-29.

DOI:10.21276/ijmrp.2016.2.6.046 